\title{
Correspondence
}

\section{Comment on "Dosimetric evaluations of the interplay effect in respiratory-gated intensity-modulated radiation therapy" [Med. Phys. 36, 893-903 (2009)]}

(Received 20 March 2009; accepted for publication 21 April 2009; published 28 May 2009)

[DOI: $10.1118 / 1.3132483$ ]

To the Editor:

In the March issue of Medical Physics, Chen et al. ${ }^{1}$ studied the dosimetric impact of the interplay between the motion of the multileaf collimator and that of a phantom undergoing simulated lung motion. Beam gating was used to limit the range of motion during radiation delivery with the goal of finding the allowable residual motion which produced "acceptable" dosimetric results. Film measurements were made in stationary and moving phantoms using simple stepand-shoot IMRT test patterns and clinical dynamic slidingwindow IMRT fields. Analysis was performed within commercial film analysis software using the gamma index ${ }^{2}$ to compare the differences between the dose distributions produced with or without motion. There are two issues of concern with this work, (1) that the gamma index is an inappropriate metric for determining "acceptability" in this situation, and (2) that the somewhat arbitrary selection of gamma analysis parameters then determines the clinical recommendation in the conclusion.

In Sec. III C, the authors stated that they adopted gamma parameters of $5 \%$ dose difference and $5 \mathrm{~mm}$ distance to agreement in order to determine which pixels "pass" and which "fail." This was done without justification for choosing these values or any statement of what values would normally be used clinically. [One can only speculate that these values were chosen by analyzing data from a nominally uniform field in Figs. 3(c) and 3(d) (solid line) and selecting parameters which made the results from this field acceptable when compared to a $10 \times 10 \mathrm{~cm}^{2}$ uniform field.] The conclusions then recommend that a residual motion window of 5 $\mathrm{mm}$ be used when selecting a gating window for clinical implementation of respiratory-gated IMRT.

As expected, any dosimetric discrepancies arising from offsets or motions less than the distance-to-agreement parameter used in the gamma analysis yield a passing result. The conclusion that a $5 \mathrm{~mm}$ gating window is acceptable is really an example of using inappropriate analysis techniques and the misuse of the gamma index as an analysis tool. The gamma index was originally intended to compare calculated and measured distributions generated under the same conditions. The technique and parameters used for analysis of the treatment delivery results should be determined carefully. Measurement of the experimental uncertainties involved in marking and registering films, and the dosimetric variability expected when making film measurements should be part of the analysis. (These arguments may also be extended to twodimensional diode or ion chamber arrays commonly used for IMRT QA measurements.) Any remaining discrepancies should then be investigated and understood as characteristics of the planning and delivery systems and the measurement tools. Examples of such characteristics are light field vs radiation field offsets due to rounded MLC leaf tips, underdosed regions due to MLC tongue and groove effect, and compromises in beam modeling within the planning system (especially in the penumbra region). All too often, it seems that the error analysis parameters are altered to cover up discrepancies that are not completely understood, or that practitioners have no ability to improve.

Though gamma analysis is often used for IMRT QA pass/ fail decisions, it is not used as a dosimetric analysis tool for this kind of experiment, especially where the spatial integrity of the experiment is a critical aspect of the experimental design. While several examples of absolute dose deviations are shown for a range of residual motions, there is no absolute characterization of these variations. This analysis does not prove that dosimetric deviations of this order are acceptable when delivering respiratory-gated IMRT. The actual magnitude and distribution of dosimetric variations should be studied within the context of the treatment plan. Whether the results are acceptable should be determined by the dosimetric criteria specified to achieve the desired clinical outcome.

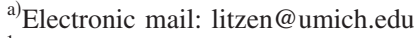

${ }^{1}$ H. Chen, A. Wu, E. D. Brandner, D. W. Heron, M. Huq, N. J. Yue, and W. Chen, "Dosimetric evaluations of the interplay effect in respiratory-gated intensity-modulated radiation therapy," Med. Phys. 36, 893-903 (2009). ${ }^{2}$ D. A. Low, W. B. Harms, S. Mutic, and J. A. Purdy, "A technique for the quantitative evaluation of dose distributions," Med. Phys. 25, 656-661 (1998).
}

D. W. Litzenberg, a) J. M. Moran, and B. A. Fraass Department of Radiation Oncology, University of Michigan, 1500 E Medical Center Drive, Ann Arbor, Michigan 48109 